\title{
Rationale Diagnostik bei Pruritus
}

Elke Weisshaar, Thomas Mettang

\begin{abstract}
Chronisches Hautjucken ist ein häufiges und bisweilen äußerst quälendes Symptom vieler Krankheiten. Nicht nur dermatologische, sondern auch viele innere, neurologische und psychische Erkrankungen können mit chronischem Pruritus einhergehen. Um zu einer raschen Diagnose zu kommen, sind eine symptomorientierte Anamnese, eine sorgfältige klinische Untersuchung und eine rationale laborchemische und apparative Diagnostik zielführend.
\end{abstract}

Demographie I Patienten aller Altersklassen sind von Pruritus betroffen. Während bei jüngeren Menschen als Ursache chronische Dermatosen wie speziell die atopische Dermatitis dominieren, müssen bei älteren Menschen systemische Erkrankungen, Medikamenteneinnahme, neurologische Veränderungen oder auch eine multifaktorielle Genese in Betracht gezogen werden [1]. Eine in Deutschland durchgeführte epidemiologische Studie belegt, dass chronischer Pruritus auch in der Allgemeinbevölkerung nicht selten ist. Nicht in jedem Fall wird das Symptom als Ausdruck einer Krankheit wahrgenommen [2, 3] und führt daher auch nicht immer zu einer ärztlichen Konsultation.

Internistische Ursachen für Pruritus I Pruritus ist eine Empfindung, die nicht nur Symptom dermatologischer, sondern auch verschiedener systemischer Erkrankungen sein kann. Aus $\triangleright$ Tab. 1 geht hervor, bei welchen internistischen Erkrankungen Pruritus auftreten kann. Darüber hinaus können auch neurologische und psychiatrische Erkrankungen mit chronischem Pruritus einhergehen.

Wegen der vielfältigen möglichen Ursachen ist die Diagnostik des chronischen Pruritus eine wichtige und herausfordernde interdisziplinäre Aufgabe.

Niereninsuffizienz I Häufig tritt Pruritus bei Patienten mit fortgeschrittener chronischer Niereninsuffizienz auf. Eine repräsentative epidemiologische Studie zeigt: 25,2\% der Dialysepatienten in Deutschland leiden zum Zeitpunkt der Untersuchung an Juckreiz, und 35,2\% haben schon vorher einmal daran gelitten, z.T. mit erheblich beeinträchtigter Lebensqualität [4-6]. Diese Studie berücksichtigt die 2007 eingeführte IFSI-Klassifikation [7] mit der Einteilung des Pruritus in drei verschiedene Klassen ( $\bullet$ Abb. 1) [8].

\section{Anamnese}

Krankheitsbefunde I Wie bei allen chronischen Krankheitszuständen ist auch bei Pruritus die Anamnese die wichtigste Grundlage, um die Ursache zu finden. Folgende Informationen sind zu erfragen:

- Vorerkrankungen und kürzlich aufgetretene Erkrankungen aller Organsysteme

- Hauterkrankung, Allergien (Kontaktallergie, Soforttyp-Allergie, Allergiepass)

- Internistische Krankheiten (Hochdruck, Stoffwechselerkrankungen, Tumore, Erkrankungen von Niere, Leber und Blutsystem)

- Neurologische Leiden

Tab. 1 Internistische Erkrankungen, die mit chronischem Pruritus assoziiert sein können.

\section{Organsystem}

Renales System

Hepatobiliäres System

Hämatopoetisches System

Endokrines System

Malassimilationssyndrome

A

Infektionskrankheiten

\section{Erkrankungen}

Chronische Niereninsuffizienz

Primär biliäre Cholangitis

Primär / sekundär sklerosierende Cholangitis

Medikamentöse / toxische Cholestase

Leberzirrhose

Obstruktive Cholestase

\section{Polycythaemia vera}

Essentielle Thrombozythämie

M. Hodgkin

Non-Hodgkin-Lymphome

Hypereosinophilie-Syndrome

Mastozytose

Diabetes mellitus

Hypothyreose, Hyperthyreose

Hyperparathyreoidismus

Karzinoidsyndrom

Fruktose- und Laktoseintoleranz

Eisenmangel

Zöliakie

Anorexia nervosa

Vitamin-B/D-Mangel

Chronische HBV-/ HCV-/ HIV-/HSV-Infektionen

VZV-Reaktivierung

Helicobacter-pylori-Infektionen

Parasitosen

Solide Tumore aller Organe 
Abb. 1 Klassifikation des chronischen Pruritus (nach International Forum for the Study of Itch, IFSI) [7]. Gruppe I: Pruritus auf primär veränderter (entzündlicher) Haut.

Gruppe II: Pruritus auf primär unveränderter (normaler) Haut.

Gruppe III: Pruritus mit Dominanz chronischer Kratzläsionen.

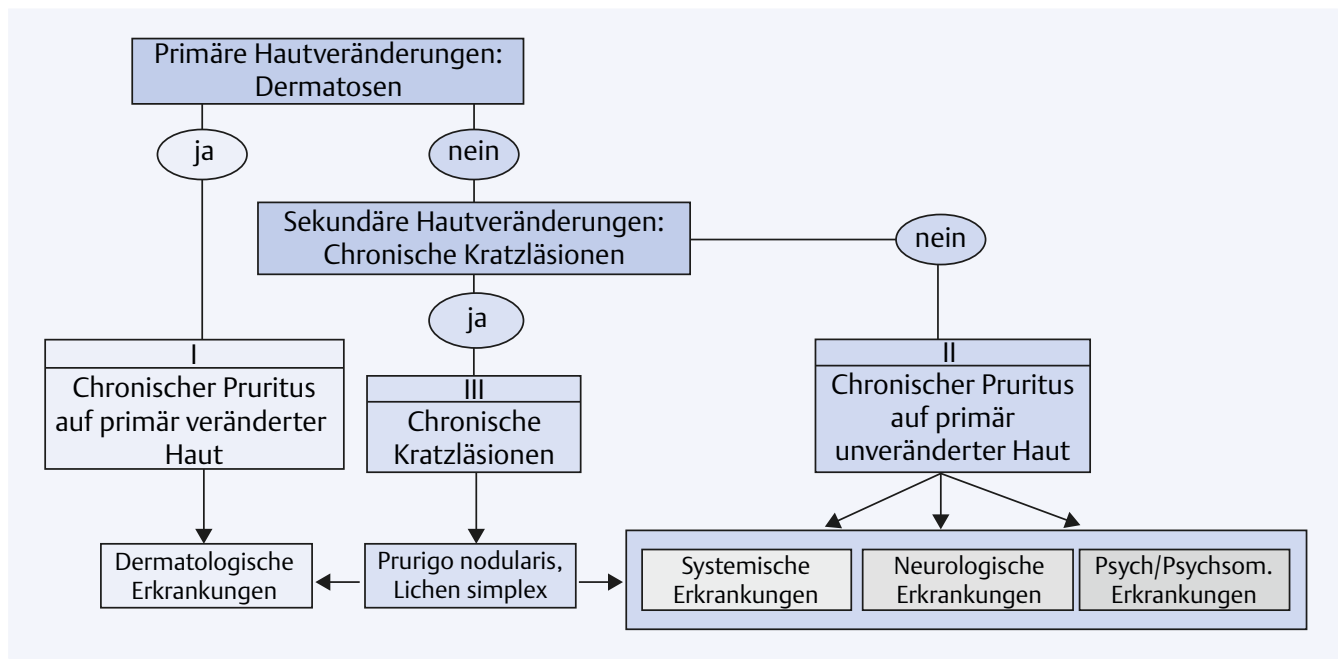

Medikamentenanamnese I Unbedingt ist eine detaillierte Medikamentenanamnese zu erheben [9].

Dabei ist es wichtig, nicht nur die aktuelle Medikation zu erfragen, sondern auch, ob sie in der Vergangenheit geändert wurde.

Interessant ist v.a. der unmittelbare Zeitraum vor Auftreten des Pruritus. Auch Bedarfsmedikamente wie z. B. Beruhigungs-, Schlaf- und Abführmitteln sollten berücksichtigt werden. Da viele Patienten auch Nahrungsergänzungsmittel, Globuli und pflanzliche Präparate zu sich nehmen, empfiehlt es sich, gezielt danach zu fragen. Patienten werten solche Produkte meist nicht als Medikamente. Weitere Informationen betreffen alle vom Patienten benutzten Salben und Cremes: möglicherweise finden sich hier wichtige Hinweise für eine bestehende Kontaktallergie. Auch die bisherigen systemischen oder lokalen Therapien zur Behandlung des Pruritus sollten hinsichtlich ihres Erfolges bzw. ihrer Unwirksamkeit abgefragt werden.

HES I Unfälle und Operationen können anamnestisch bedeutsam sein, besonders, wenn sie sich vor der Manifestation des Pruritus ereigneten bzw. stattfanden. So kann z. B. auch zur Volumensubstitution applizierte Hydroxyethylstärke (HES) zu chronischem Pruritus führen.

Vegetative Anamnese I Im Rahmen der vegetativen Anamnese ist der Patient gezielt nach begleitenden Beschwerden wie Müdigkeit, Schlafstörungen, Gewichtsverlust, Fieber, Nachtschweiß, Unruhe, Stress und Nervosität zu befragen.

Neuropathischer Pruritus I In jüngster Zeit gibt es Hinweise darauf, dass chronische Affektionen der Wirbelsäule den sogenannten neuropathischen Pruritus induzieren können. Darum sollte bei entsprechender Lokalisation des Pruritus gezielt nach Schmerzen oder Beschwerden im Rücken oder Nacken gefragt werden. Begleitsensationen wie Brennen, Stechen, Prickeln, Beißen und Ameisenlaufen können auf mögliche neuropathische Komponenten bzw. auf eine Polyneuropathie hindeuten.

Abdominale Beschwerden I Oberbauchbeschwerden können Anzeichen für eine Gastritis, ein Ulcus ventriculi oder ein Ulcus duodeni sein. Eine Helicobacter-pylori-Infektion kann für den chronischen Pruritus pathogenetisch relevant sein.

Psychosoziale Anamnese I Gerade bei älteren Menschen sollten Veränderungen im sozialen Umfeld gezielt erfasst werden. So ist z. B. zu erfragen, ob eine Kurzzeitpflege im Heim stattgefunden hat oder ob die Pflege durch Angehörige erfolgt. Falls nötig empfiehlt es sich, einen Fragebogen einzusetzen oder Angehörige mit einzubeziehen. Auch die Exploration des psychosozialen Befindens des Patienten ist im Rahmen der Anamnese bedeutsam.

Bei Verdacht auf eine psychiatrische oder psychosomatische Erkrankung sollte der Patient fachärztlich untersucht werden.

Schwere psychiatrische Erkrankungen als Ursache für Pruritus sind selten (Dermatozoenwahn). Bei einer Reihe von Patienten spielen psychische Faktoren eine erhebliche Rolle für die Auslösung oder Ausprägung des Pruritus. Auch somatoformer Pruritus kann beobachtet werden.

Zeitliche Aspekte I Allgemein ist zu sagen, dass ein plötzlicher Beginn des Hautjuckens bei ansonsten unauffälliger Pruritusanamnese Hinweis auf ein allergisches Kontaktekzem, eine Urtikaria, eine Arzneimittelreaktion oder eine Scabies sein kann. Ein langsamer Beginn entspricht häufiger einer systemischen Ursache oder ist Folge einer schon länger bestehenden Hautrockenheit (Xerosis cutis). Besonders wichtig ist die Erfassung des exakten Zeitpunktes, wann der Pruritus begonnen hat. Häufig können Patienten sehr ge- 
nau Zusammenhänge oder Fakten beschreiben, die mit dem Beginn der Beschwerden korrelieren (z.B. ein Medikamentenwechsel). Auch der zeitliche Verlauf des Pruritus ist zu erfragen, z.B. ob er immer gleich stark ist, tageszeitlich bedingt schwankt oder anderen Einflüssen unterliegt.

Lokalisation allgemein I In der Vergangenheit ging man davon aus, dass generalisierter Pruritus in der Regel systemisch bedingt ist. Dies konnte so nicht bestätigt werden [10]. Zwar ist bei Dermatosen (z.B. atopisches Ekzem, Psoriasis) der Pruritus häufiger auf die entzündeten Hautstellen begrenzt, aber auch hier gibt es viele Patienten mit generalisiertem Pruritus. Insofern sollte stets danach gefragt werden, welche Hautstellen betroffen sind. Oft kann die Lokalisation des Pruritus wichtige differentialdiagnostische Hinweise geben. Man muss jedoch berücksichtigen, dass auch im Falle eines initial lokalisierten, z. B. neuropathischen Pruritus eine sekundäre Generalisierung möglich ist [12].

Pruritus im Genitoanalbereich I Von anamnestischem Interesse sind Erkrankungen des Darmes, insbesondere Hämorrhoidalleiden, eingesetzte Kosmetika, gynäkologische und urologische Vorgeschichte und sexuelle Besonderheiten. Auch chronische Rückenleiden sind zu berücksichtigen, da diese neben Schmerzen auch Juckempfindungen im Analbereich hervorrufen können [11].

Patientensicht einbeziehen I Stets ist der Patient bezüglich seiner eigenen Theorie zur Ursache des Juckreizes zu befragen. So könnte vermehrtes Hautjucken durch Wasserkontakt beim Duschen oder Baden auf einen aquagenen Pruritus hinweisen. Dieser tritt häufig bei Xerosis cutis auf, ist aber auch stets Anlass für die Abklärung einer hämatologischen Ursache. Viele Patienten haben ihre eigene Methode, den Juckreiz zu mindern. Auch danach sollte gezielt gefragt werden. So kühlen Patienten mit neuropathischem Pruritus ihre Haut, z.B. mit kalten Waschlappen, kalten Duschen oder mit der Auflage von Eisbeuteln.

\section{Klinische Befunde}

Körperliche Untersuchung I Um die Ursache für einen chronischen Pruritus zu finden, darf man sich keinesfalls auf die Inspektion einzelner Körperareale beschränken.

Es sollte die gesamte Haut inklusive Schleimhäute, Kopfhaut, Haare, Nägel und Genitoanalregion untersucht werden.

Typisch ist das „Schmetterlingszeichen“: Am gesamten Rücken finden sich Kratzläsionen - bis auf eine Aussparung in der Mitte zwischen den Schulterblättern ( $\bullet$ Abb. 2 ), weil der Betroffene

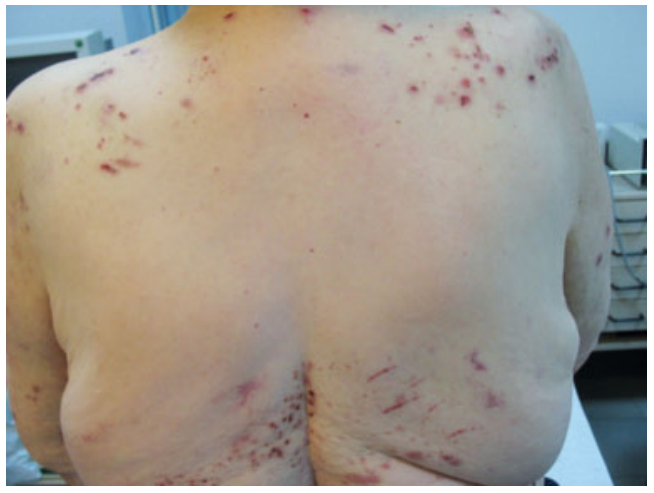

Abb. 2 Sogenanntes "Schmetterlingszeichen“ am Rücken einer Patientin mit chronischem Pruritus bei dialysepflichtiger Niereninsuffizienz.

dort nicht mit den Händen hingelangt. Im Rahmen der allgemeinen körperlichen Untersuchung müssen auch die Lymphknoten beurteilt werden. Zudem sollte auf Körpergeruch geachtet werden, wie er bei Leber- und Nierenkranken oft in typischer Weise vorkommt. Die Färbung der Haut und der Skleren kann Hinweise auf einen Ikterus oder eine Anämie geben $(\bullet$ Abb. 3 ).

Prurigo nodularis I Wenn die Haut Knoten und Knötchen aufweist, kann eine sogenannten Prurigo nodularis (IFSI-Klasse III) vorliegen. Die Ätiologie ist in vielen Fällen gemischt oder systemisch bedingt. Eine Prurigo nodularis tritt häufig bei atopischer Dermatitis oder ausgeprägter atopischer Hautdiathese auf.

Kratzspuren I Nicht selten setzen Patienten zum Kratzen Gegenstände (z.B. Bürsten und Stifte) ein, die entsprechende Schädigungsmuster bzw. Kratzspuren auf der Haut hinterlassen. Um die Hautbefunde besser einordnen zu können, muss auch das Kratzverhalten der Patienten gezielt erfragt werden. So macht es z. B. einen Unterschied, ob der Patient kratzt, reibt oder die juckenden Stellen anderweitig manipuliert.

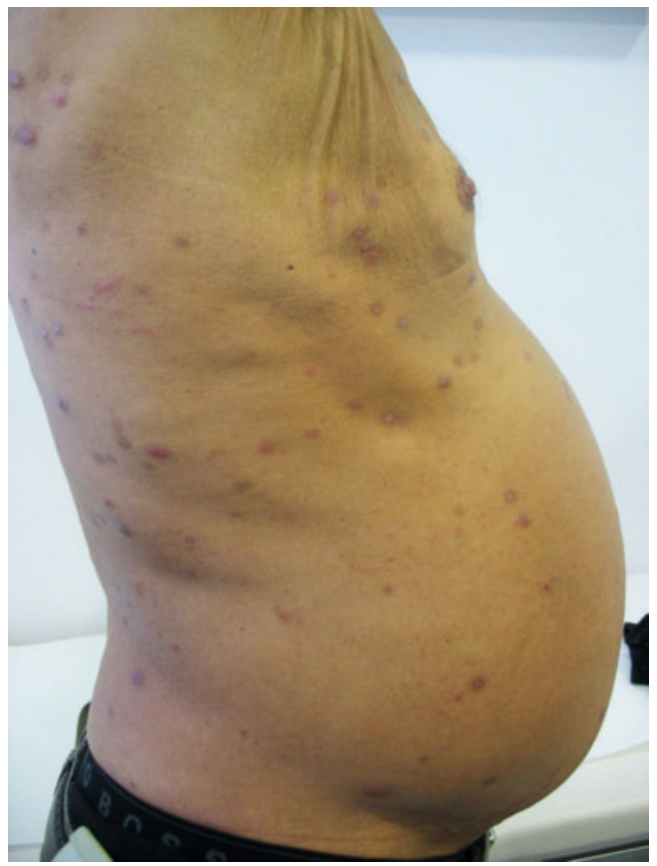

Abb. 3 Hautveränderungen bei cholestatischem Pruritus. Ikterus sowie hyperkeratotische, z. T. exkoriierte Knoten im Sinne einer Prurigo nodularis. (Der Bauch ist durch einen mäßigen Aszites leicht vorgewölbt.) 


\section{Befund}

Analer Pruritus

Aquagener und genitaler Pruritus, Pruritus unklarer Genese

Blutbildveränderungen, Verdacht auf lymphoproliferative Erkrankungen

Eisenmangel, Stuhlunregelmäßigkeiten Pathologische Leberwerte

Pathologische Nüchternglukose

Primäre oder sekundäre Hautveränderungen

\section{V.a. Allergie}

V.a. endokrine Erkrankungen

\section{V.a. HIV}

V.a. Mastozytose

V.a. neuroendokrine Tumore

V.a. Porphyrien

V.a. neuroendokrine

Tumore

V.a. Mastozytose

\section{Untersuchung}

Parasiten, Wurmeier

digital-rektale Untersuchung

PSA

Laktose-/ Sorbit-Intoleranztest

Vitamin $\mathrm{B}_{12}$

Folsäure

Eiweißelektrophorese

Immunfixation

JAK2-Status Durchflusszytometrie ggf. KM-Punktion

Stuhluntersuchung auf okkultes Blut

Hepatitisserologie

- anti-HVA-IgM

- HBsAg,

- anti-HBc,

- anti-HCV

Gallensäuren

AMA (antimitochondriale Antikörper)

pANCA (perinukleäre antineutrophile ytoplasmatische Antikörper)

ANA (antinukleäre Antikörper)

SMA (glatte Muskulatur-Antikörper)

SLA (Iösliches Leberantigen-Antikörper)

LKM (Leber-Nieren-Mikrosomen-Antikörper)

Gewebstransglutaminase-AK

Alpha-Fetoprotein (bei Leberzirrhose/

hepatischer Raumforderung)

$\mathrm{HbA}_{1 \mathrm{c}}$

Glukose-Toleranztest

Direkte und indirekte Immunfluoreszenz Auto-Antikörper gegen dermale Proteine (BP 180, 230, Desmoglein)

Gesamt-IgE, ggf. spezifische lgE

Prick-Test

Epikutantest

Parathormon

fT3, fT4

Phosphat, $\mathrm{Ca}^{2+}$

25-OH-Cholecalciferol

TRAK (TSH-Rezeptor-AK)

TPO-AK (Thyreoperoxidase-AK)

HIV-Serologie, ggf. Lues-Serologie

Tryptase

Chromogranin A

24h-Sammelurin:

- Porphyrine

- 5-Hydroxyindolessigsäure

- Methylimidazolessigsäure

Tab. 2 Fakultative weitergehende Labordiagnostik entsprechend der klinischen, chemischen und apparativen Vorbefunde (angelehnt an die S2-Leitlinien Pruritus 2016 [8]).

Effloreszenzen I Auch gering ausgeprägte Effloreszenzen können einer (chronischen) Dermatose entsprechen. Daher sollte in diesen Fällen ein Dermatologe hinzugezogen werden. Hautkolorit, Morphologie und Verteilungsmuster der Effloreszenzen sowie mögliche Hautzeichen einer systemischen Erkrankung sind zu dokumentieren Primäre und sekundäre, also durch Kratzen verursachte Effloreszenzen wie z.B. Exkoriationen, Erosionen, Krusten oder Petechien müssen erfasst und unterschieden werden $(\checkmark$ Abb. 4$)$.

\section{Labordiagnostik bei Patienten ohne primäre Hautveränderungen}

Neue Leitlinien I Die S2-Leitlinien zu Diagnostik und Therapie des chronischen Pruritus wurden aktuell überarbeitet. Die Neufassung gibt vor, wie bei Patienten ohne entsprechende Vorinformation oder Hinweise auf eine bestehende innere oder neurologisch-psychiatrische Erkrankung zu verfahren ist [8]. Empfohlen wird folgende Labordiagnostik:

- Bilirubin, Transaminasen (GPT [ALAT], GOT [ASAT]), Gamma-Glutamyl-Transferase (GGT, Gamma-GT), alkalische Phosphatase

- Blutbild mit Differenzialblutbild, Ferritin

- Blutsenkungsgeschwindigkeit (BSG) und C-reaktives Protein (CRP)

- Blutzucker nüchtern

- Kreatinin, Harnstoff, errechnete glomeruläre Filtrationsrate (eGFR), $\mathrm{K}^{+}$, Urin (Streifentest)

- Laktatdehydrogenase (LDH)

- Thyreoidea-stimulierendes Hormon (TSH)

Anhand dieser Parameter gewinnt der Arzt erste Hinweise auf evtl. bestehende innere Krankheiten (z.B. Niereninsuffizienz, Lebererkrankungen, Störungen im blutbildenden System). Bei auffälligen Befunden schließen sich differenzialdiagnostische Untersuchungen an ( $\bullet$ Tab. 2). Je nach Entität ist eine spezifische fachärztliche Weiterbehandlung erforderlich.

Einem chronischen Pruritus ohne Hautveränderungen liegt häufig eine der drei folgenden Erkrankungen zugrunde: Eisenmangel, Niereninsuffizienz und Lebererkrankungen.

Abb. 4 Typische Hautveränderungen bei urämischem Pruritus. Kratzspuren am Bauch mit Exkoriationen.

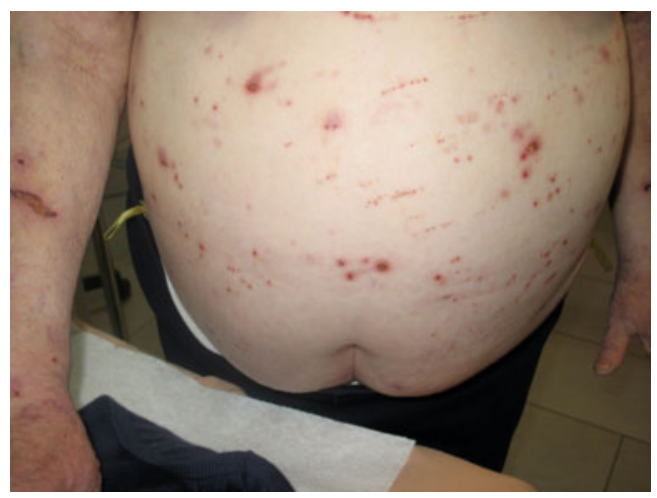


Eisenmangel I Eisenmangel führt nicht selten zu genitoanalem, aber auch generalisiertem Pruritus. Üblicherweise sind bei Eisenmangel das mittlere korpuskuläre Volumen (MCV), das mittlere korpuskuläre Hämoglobin $(\mathrm{MCH})$ und das Serum-Hämoglobin vermindert. Die „Überlagerung" durch andere Erkrankungen wie

- Vitamin-B ${ }_{12}$-Mangel,

- Folsäuremangel oder

- myelodysplastisches Syndrom

kann die Ergebnisse der o.g. Indizes verfälschen. Auch wenn das Blutbild keine Mikrozytose zeigt, kann trotzdem ein funktioneller Eisenmangel bestehen, z. B. bei fortgeschrittener Niereninsuffizienz. Andererseits gibt es Krankheitsbilder wie die Thalassämie, wo eine Mikrozytose vorliegt, die Eisenwerte aber im Normbereich sind. Bei unklaren Befunden ist die Analyse der Eisenspeicherparameter oft hilfreich. Doch auch die Bestimmung von Ferritin und Transferrinsättigung führt nicht immer zu einem eindeutigen Befund. Denn Ferritin kann als sog. Akutphasenprotein im Rahmen einer Leberzirrhose, einer Tumorerkrankung oder einer Infektion erhöht sein, obwohl ein Eisenmangel besteht $[13,14]$. Hier hilft ggf. die Bestimmung von hypochromen Erythrozyten und Retikulozyten-Hämoglobin.

Niereninsuffizienz I Kreatinin ist als Abbauprodukt des Muskelstoffwechsels einfach im Serum nachweisbar. Je nach Analysemethode und Labor ergeben sich unterschiedliche Normalwerte. Allerdings kann sich auch hinter einem „normalen“ Serum-Kreatinin eine bereits erheblich eingeschränkte Nierenfunktion im Sinne einer verminderten glomerulären Filtrationsrate verstecken ( $\triangleright$ Abb. 5). Abhängig von Muskelmasse, Muskelumsatz und Muskelstoffwechsel ist bei eingeschränkter Nierenfunktion das Serum-Kreatinin mehr oder weniger erhöht.

- So hat z.B. ein junger muskelkräftiger Mann mit grenzwertig erhöhtem Serum-Kreatinin von $1,5 \mathrm{mg} / \mathrm{dl}$ u.U. eine normale Nierenfunktion.

- Hingegen kann bei einer älteren, immobilen Patientin mit gleich hohem Serum-Kreatinin eine Einschränkung der glomerulären Filtrationsrate von $60-70 \%$ vorliegen.

Um die Nierenfunktion besser einschätzen zu können, gibt es verschiedene Vorgehensweisen. Zum einen besteht die Möglichkeit, die Kreatinin-Clearance zu bestimmen (cave: Sammelfehler!). Zum anderen kann eine der gebräuchlichen Formeln zur Abschätzung der GFR (eGFR mittels MDRD oder CKD-EPI-Formel) zum Einsatz kommen. Alter und Geschlecht des Patienten fließen in beide Formeln mit ein.

Lebererkrankungen I Bereits Jahre, bevor der Bilirubinwert erhöht ist, kann bei der primären biliären Cholangitis Pruritus auftreten. Allerdings sind zu diesem Zeitpunkt meistens bereits alkalische Phosphatase, $\gamma$-GT sowie immunologische Parameter wie antinukleäre und antimitochond-

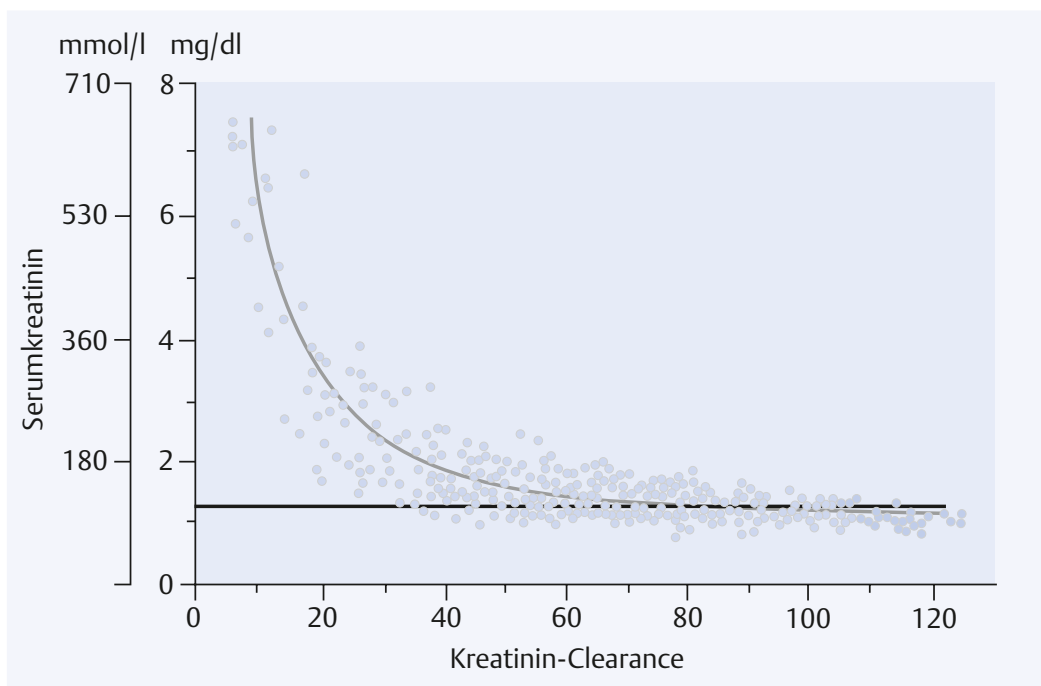

Abb. 5 Zusammenhang zwischen Serum-Kreatinin und glomerulärer Filtrationsrate (GFR) (Bildnachweis: Kuhlmann et al. Nephrologie. 6. Auflage 2015. Thieme Stuttgart, New York). nach Krankheitsbeginn nachweisbar. Im Zweifel ist die Hepatitis-C-RNA zu bestimmen.

Bei den meisten Lebererkrankungen, die mit Pruritus einhergehen, finden sich entweder erhöhte Transaminasen, erhöhtes Bilirubin und / oder erhöhte Cholestaseparameter (alkalische Phosphatase, y-GT).

\section{Weiterführende Untersuchungen}

Spezielle dermatologische Untersuchungen I Bei Verdacht auf eine spezifische Dermatose ist ggf. eine Probebiopsie der Haut mit dermatohistologischer Untersuchung durchzuführen. Besteht eine Scabies schon lange Zeit und / oder wurde diese lokal mit Kortison behandelt, kann dies ein Ekzembild imitieren. Dieser Befund ist daher ebenfalls dermatohistologisch abzuklären. Bei Verdacht auf eine Epizoonose kann die dermatoskopische Untersuchung helfen, z.B. Milbengänge zu erkennen. Da starker Pruritus auch bei blasenbildenden Immundermatosen unter dem Bild von Exkoriationen auftreten kann, sollte man die Diagnose durch eine direkte Immunfluoreszenz (DIF) absichern. Ein HES-induzierter Pruritus kann nur durch eine elektronenmikroskopische Untersuchung der Haut diagnostiziert werden. Besteht der Verdacht auf ein allergisches Kontaktekzem, sind Epikutantests durchzuführen. Bei Atopie und Verdacht auf eine Soforttyp-Allergie sind entsprechende Pricktests und eine In-vitro-Diagnostik mit Gesamt-IgE und entsprechenden IgE-Bestimmungen sinnvoll.

\footnotetext{
Abhängig von Hautbefunden und Differentialdiagnosen sind bakteriologische und mykologische Untersuchungen zu veranlassen.
} 


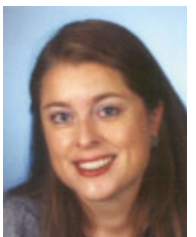

Prof. Dr. med. Elke Weisshaar

ist stellvertretende ärztliche Direktorin der Abteilung Klinische Sozialmedizin, Schwerpunkt: Berufs- und Umweltdermatologie, Universität Heidelberg.e elke.weisshaar@ med.uni-heidelberg.de

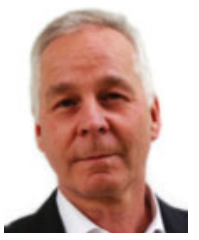

Prof. Dr. Thomas Mettang ist leitender Arzt an der DKD Helios Klinik und am KfH-Nierenzentrum Wiesbaden mettang@ nephrologie-wiesbaden.de

Interessenkonflikt

E. W. erklärt, dass keine Interessenkonflikte bestehen. T. M. erklärt, dass er Vortragshonorare von FMC erhalten hat.

DOI 10.1055/s-0042-114092

Dtsch Med Wochenschr 2016; 141: 1711-1716

(c) Georg Thieme Verlag KG . Stuttgart · New York .

ISSN 0012-0472
Bildgebende Verfahren I Bei chronischem Pruritus unklarer Genese beschränkt man sich nach den Laboruntersuchungen im ersten Ansatz auf eine Sonographie des Bauchraumes und ein Röntgenbild des Thorax. Mit Hilfe dieser Verfahren sollen Hinweise auf eine lymphoproliferative oder maligne Erkrankung abgeklärt werden. Spezifische klinische oder laborchemische Befunde (z.B. Hinweise für eine maligne Erkrankung) können weitere apparative Untersuchungen erforderlich machen. Zur weiteren Einordnung von Nieren- oder Lebererkrankungen kommen Sonographie, Szintigraphie und Schnittbildtechniken (CT, MRT) zum Einsatz. Bei analem Pruritus sollte stets eine Proktoskopie, ggf. auch Rektoskopie erfolgen, bei genitalem Pruritus ist eine gynäkologische bzw. urologische Untersuchung indiziert. Bei Verdacht auf neuropathischen Pruritus bei Patienten mit chronischen Rückenschmerzen und/oder entsprechender Anamnese ist ein MRT anzufertigen.

Konsequenz für Klinik und Praxis

- Chronischer Pruritus ist ein häufiges Symptom. Er beeinträchtigt die Lebensqualität stark und bedarf einer sorgfältigen ärztlichen Abklärung.

- Chronischer Pruritus kann alle Altersgruppen betreffen und Ausdruck einer dermatologischen, allergologischen, internistischen, neurologischen oder psychiatrischen Erkrankung sein.

- Chronischer Pruritus kann als Symptom der Diagnose einer inneren Erkrankung vorausgehen.

- Chronischer Pruritus hat häufig eine multifaktorielle Genese und muss interdisziplinär abgeklärt werden.

- Zur umfassenden Anamnese bei chronischem Pruritus gehört es, alle aktuell und zuvor verwendeten Präparate sorgfältig zu erfassen - auch Medikamente, die im Rahmen von Operationen verabreicht wurden.

- Aufgrund der demographischen Entwicklung und der Zunahme von chronischem Juckreiz bei älteren Menschen werden Allgemeinärzte und Internisten immer häufiger damit konfrontiert sein.

- Auch unter Einsatz aller diagnostischen Möglichkeiten ist es oft nicht möglich, die zugrundeliegende Erkrankung zu eruieren $[15,16]$. In diesem Fall empfiehlt sich - bei Persistenz des Hautjuckens - eine Wiederholung der Untersuchung nach 12 Monaten.

\section{Literatur}

1 Weisshaar E, Dalgard F. Epidemiology of itch: adding to the burden of skin morbidity. Acta Derm Venereol 2009; 89 (4): $339-350$

2 Matterne U, Apfelbacher C], Vogelgsang L et al. Incidence and determinants of chronic pruritus: a population-based cohort study. Acta Derm Venereol 2013; 93 (5): 532 - 537

3 Matterne U, Apfelbacher C], Loerbroks A et al. Prevalence, correlates and characteristics of chronic pruritus: a population-based cross-sectional study. Acta Derm Venereol 2011; 91 (6): 674-679

4 Weiss M, Mettang T, Tschulena U, Weisshaar E. I. Health-related quality of life in haemodialysis patients suffering from chronic itch: results from GEHIS (German Epidemiology Haemodialysis Itch Study) Qual Life Res. 2016 ]un 15. [Epub ahead of print]

5 Hayani K, Weiss M, Weisshaar E. Clinical Findings and Provision of Care in Haemodialysis Patients with Chronic Itch: New Results from the German Epidemiological Haemodialysis Itch Study. Acta Derm Venereol 2016; 96: 361 - 366

6 Weiss M, Mettang T, Tschulena U et al. Prevalence of chronic itch and associated factors in haemodialysis patients: a representative cross-sectional study. Acta DermVenereol 2015; 95 (7): 816 - 821

7 Ständer S, Weisshaar E, Mettang T et al. Clinical classification of itch: a position paper of the International Forum for the Study of Itch. Acta Derm Venereol 2007; 87 (4): 291 - 294

8 Ständer, S., Zeidler C, Augustin M et al. S2k Leitlinie zu Diagnostik und Therapie des chronischen Pruritus. AWMF 2016 im Druck

9 Maleki K, Weisshaar E. Arzneimittelinduzierter Pruritus. Hautarzt 2014; 65 (5): 436-442

10 Weisshaar E, Apfelbacher C, Jager G et al. Pruritus as a leading symptom: clinical characteristics and quality of life in German and Ugandan patients. $\mathrm{Br}$ J Dermatol 2006; 155 (5): 957 - 964

11 Weisshaar E. Genitoanaler Pruritus. Hautarzt 2015; 66(1): $53-59$

12 Kwatra SG, Stander S, Bernhard JD et al. Brachioradial pruritus: a trigger for generalization of itch. J Am Acad Dermatol 2013; 68 (5): 870 - 873

13 Piedras J, Alvarez E, Herrera FM et al. Clinical usefulness of mean corpuscular volume and red cell distribution width in iron deficient blood donors. Rev Invest Clin 1993; 45 (5): 469-472

14 Kis AM, Carnes M. Detecting iron deficiency in anemic patients with concomitant medical problems. J Gen Intern Med 1998; 13 (7): 455 - 461

15 Weisshaar E, Weiss M, Mettang T et al. Paraneoplastic itch: an expert position statement from the Special Interest Group (SIG) of the International Forum on the Study of Itch (IFSI). Acta DermVenereol 2015; 95 (3): 261 - 265

16 Sommer F, Hensen P, Bockenholt B et al. Underlying diseases and co-factors in patients with severe chronic pruritus: a 3-year retrospective study. Acta Derm Venereol 2007; 87 (6): 510 - 516 